\title{
Antibiotic susceptibility pattern and risk factors associated with Acinetobacter and Pseudomonas infection at a tertiary care hospital
}

Sherchan JB iD

Jatan Bahadur Sherchan, Associate Professor, Department of Microbiology, Kathmandu University School of Medical Sciences, Dhulikhel, Kavrepalanchok, Nepal.

\begin{abstract}
Background: Infection due to Acinetobacter spp. and Pseudomonas aeruginosa is a major worldwide concern these days. Antibiotic resistance and predisposing factors among the patients for acquiring such infection is a major challenge globally and in Nepal.

Objectives: To determine antimicrobial susceptibility pattern of Acinetobacter spp. and Pseudomonas aeruginosa isolates along with predisposing factors.

Methods: A total of 9,705 clinical samples were processed in this analytical cross-sectional study from December 2019 to November 2020. Antibiotic susceptibility pattern was determined following Clinical Laboratory Standard Institute guidelines. Patients' information was obtained after informed consent.

Results: Acinetobacter spp. and Pseudomonas aeruginosa isolates were 92 (0.95\%). Fifty-three (57.61\%) samples were respiratory samples. Thirteen (20\%) Pseudomonas aeruginosa and 18 (66.67\%) Acinetobacter spp. were multidrugresistant (MDR). Eight (12.31\%) Pseudomonas aeruginosa strains and 13 (48.15\%) Acinetobacter spp. strains were sensitive only to Colistin. Twenty-two (95.65\%) prolonged hospital stayers had MDR bacteria compared to only nine (13.04\%) non-prolonged hospital stayers ( $p$-value $<0.001)$. Sixteen $(94.12 \%)$ of diabetic patients had MDR bacteria isolates in comparison to only $15(20 \%)$ of non-diabetic patients ( $p$-value $<0.001)$. Thirty-one $(33.69 \%)$ were elderly patients (age $\geq 65$ years) and 61 (66.31\%) were of age less than 65 years old. Seventeen (54.84\%) of elderly patients had MDR isolates whereas only 14 (22.95\%) of patients who are not elderly had MDR isolates ( $p$-value $=0.0047$ ).

Conclusion: Acinetobacter spp. and Pseudomonas aeruginosa strain were isolated from various samples. For effective treatment of infection by such organisms detailed microbiological diagnosis and drug susceptibility testing is needed along with identification of predisposing factors.
\end{abstract}

Key words: Acinetobacter spp.; Multidrug resistant; Pseudomonas aeruginosa.

\section{Access this article online}

Website: www.jkmc.com.np

DOI: https://doi.org/10.3126/jkmc.v10i1.38945

\section{HOW TO CITE}

Sherchan JB. Antibiotic susceptibility pattern and risk factors associated with Acinetobacter and Pseudomonas infection at a tertiary care hospital. J Kathmandu Med Coll. 2021;10(1):4-10.

\section{Address for correspondence}

Dr. Jatan Bahadur Sherchan,

Associate Professor, Department of Microbiology

Kathmandu University School of Medical Sciences (KUSMS),

Dhulikhel, Kavrepalanchok, Nepal

Email: jatansherchan@gmail.com

Copyright @ 2021 Journal of Kathmandu Medical College (JKMC) ISSN: 2019-1785 (Print), 2091-1793 (Online)

\section{INTRODUCTION}

Tnfections caused by drug resistant Acinetobacter spp. Land Pseudomonas aeruginosa are emerging cause of Hospital Acquired Infection and a significant threat to public health.' These bacteria cause a wide spectrum of infections that include pneumonia, bacteraemia, meningitis, urinary tract infection and wound infection. Recent studies done in western and central region of Nepal revealed that the infections caused by drug resistant Acinetobacter spp. and Pseudomonas aeruginosa are associated with prolonged hospital stay and mortality. ${ }^{2,3}$

Globally, Acinetobacter spp. and Pseudomonas aeruginosa has also been identified as an ESKAPE pathogen (Enterococcus faecium, Staphylococcus aureus, Klebsiella pneumoniae, Acinetobacter baumannii, Pseudomonas 
aeruginosa, and Enterobacter species). The ESKAPE pathogens are a group of pathogens with high rate of antibiotic resistance that are responsible for the majority of nosocomial infections. ${ }^{4}$

In such situations it is very necessary to conduct research studies on antimicrobial susceptibility of these pathogens along with associated predisposing factors. Hence, this study aims to find out antibiotic sensitivity patterns along with few predisposing factors associated with Acinetobacter spp. and Pseudomonas aeruginosa infections.

\section{METHODOLOGY}

The study was an analytical cross-sectional study, which was conducted at Microbiology lab of Kathmandu University Hospital, Dhulikhel from the month of December 2019 to November 2020. Ethical approval was taken from the Institutional Review Committee of Kathmandu University Hospital before the study was conducted (Ref. 254/19).

Any clinical sample (sputum, tracheal aspirate, endotracheal secretion, pus, wound swab, blood, urine and other body fluids) from which Acinetobacter spp. or $P$. aeruginosa was isolated were only included in the study excluding other bacteria. The sample was processed for culture and sensitivity as recommended by Clinical Laboratory Standards Institute (CLSI) $2018 .^{5}$

Informed consent was taken from the patients from whom Acinetobacter spp. or P. aeruginosa isolates were detected and clinical information was obtained by history taking and clinical examination by the researcher himself with the help of consultant doctor taking care of the patient as well as by going through the medical and lab records.

The samples were cultured on 5\% sheep blood agar (BA) and MacConkey agar (MA) plates. The BA and MA plates were incubated at $37^{\circ} \mathrm{C}$ for 24 hours in an aerobic atmosphere. All the bacteria were isolated and identified using colony morphology, microscopy, and biochemical tests following standard procedures. ${ }^{5}$

The isolates were tested for antibiotic susceptibility by modified Kirby-Bauer disc diffusion method in compliance with CLSI 2018 guidelines on Mueller-Hinton agar (MHA) plates. ${ }^{5}$ All the isolates of Acinetobacter spp. were investigated for antimicrobial susceptibility testing (AST) against ciprofloxacin $(5 \mu \mathrm{g})$, gentamicin $(10 \mu \mathrm{g})$, amikacin $(30 \mu \mathrm{g})$, cefepime $(30 \mu \mathrm{g})$, cefotaxime $(30 \mu \mathrm{g})$, cefoperazone-sulbactam $(75 / 30 \mu \mathrm{g})$, levofloxacin $(5 \mu \mathrm{g})$, gentamicin $(10 \mu \mathrm{g})$, imipenem $(10 \mu \mathrm{g})$, meropenem $(10$ $\mu \mathrm{g})$, piperacillin/tazobactam $(100 / 10 \mu \mathrm{g})$ using Hi-Media India Pvt. Ltd following the Kirby-Bauer method on Mueller-Hinton agar. A suspension of the test organism was prepared in peptone water and matched to 0.5 McFarland standard for AST. With the help of a sterile cotton swab, lawn culture of the suspension was made on a Mueller-Hinton agar plate. Three MHA plates were used and the antibiotic discs were placed on MHA maintaining a $25 \mathrm{~mm}$ distance between two discs and were incubated at $37^{\circ} \mathrm{C}$ for 24 hours. After 24 hours, the zone of inhibition was measured for each antibiotic, and results were interpreted as sensitive, intermediate, and resistant on the basis of CLSI guidelines, 2018. ${ }^{5}$ For Colistin susceptibility broth microdilution (BMD) method was done according to CLSI 2018 guidelines in which a susceptibility breakpoint is $\leq 2 \mathrm{mg} /$ liter. ${ }^{5}$ Escherichia coli ATCC 25922 and Pseudomonas aeruginosa ATCC 27853 were used as the control organisms for antibiotic sensitivity.

Definition of Multidrug-resistant Acinetobacter: Any Acinetobacter spp. that has tested either Intermediate (I) or Resistant (R) to at least one drug in at least three of the following six categories:

1. Extended-spectrum cephalosporin (cefepime, ceftazidime, ceftriaxone, cefotaxime)

2. Fluoroquinolones (ciprofloxacin, levofloxacin)

3. Aminoglycosides (amikacin, gentamicin, tobramycin)

4. Carbapenems (imipenem, meropenem, doripenem)

5. Piperacillin/tazobactam 6. Ampicillin/sulbactam. ${ }^{6}$

Definition of Multidrug-resistant Pseudomonas aeruginosa: Pseudomonas aeruginosa that has tested either Intermediate (I) or Resistant (R) to at least one drug in at least three of the following five categories:

1. Extended-spectrum cephalosporin (cefepime, ceftolozane/tazobactam, ceftazidime/avibactam)

2. Fluoroquinolones (ciprofloxacin, levofloxacin)

3. Aminoglycosides (amikacin, gentamicin, tobramycin)

4. Carbapenems (imipenem, meropenem, doripenem)

5. Piperacillin/tazobactam. ${ }^{6}$

Prolonged hospitalisation was defined as length of stay longer than two weeks. ${ }^{7}$ Patients of age 65 years or older was considered as elderly person. ${ }^{8}$

Data were analysed by SPSS Statistics for Windows, version 18.0 (SPSS Inc., Chicago, III., USA) and p-value $<0.05$ was considered significant. 


\section{RESULTS}

Total number of samples (respiratory sample, pus, wound swab, urine, blood, other body fluids) received during the study period was 9,705 . Out of this Acinetobacter spp. and $P$. aeruginosa isolates were 92 (0.95\%). Most of the samples from which isolates were found were of respiratory tract origin which was 53 (57.61\%). Sixteen (59.26\%) of Acinetobacter spp. isolates and 37 (56.92\%) if $P$. aeruginosa isolates were detected in the respiratory sample and the total number of Acinetobacter spp. isolates was $27(29.35 \%)$ and $P$. aeruginosa isolates was 65 (70.65\%). Table 1 as shown below provides the number of each type of clinical sample received along with Acinetobacter spp. and $P$. aeruginosa isolates from the sample during the study period.

Antibiotic susceptibility pattern of $P$. aeruginosa showed that, $52(80 \%)$ of the strains were sensitive to Ciprofloxacin, Ceftazidime, and Cefepime; 53(81.54\%) were sensitive to Levofloxacin; 55 (84.61\%) were sensitive to Piperacillin-tazobactum, Gentamicin, and Amikacin; 57 (87.69\%) were sensitive to carbapenem; and all strains were sensitive to Colistin as shown in Table 2.

Antibiotic susceptibility pattern of Acinetobacter spp. showed that, six (22.22\%) of the strains were sensitive to Gentamicin and Amikacin; nine (33.33\%) were sensitive to Ciprofloxacin, Levofloxacin Ceftazidime, Cefotaxime, Cefepime; 10 (37.04\%) were sensitive to Cefoperazonesalbactum and Piperacillin-tazobactum; 14 (51.85\%) were sensitive to carbapenem and all strains were sensitive to Colistin (Table 2 ).

Thirteen (20\%) P. aeruginosa and 18 (66.67\%) Acinetobacter spp. were multidrug-resistant. Over all 31 (33.69\%) of the isolates were multidrug-resistant.

Eight (12.31\%) P. aeruginosa and 13 (48.15\%) Acinetobacter spp. were sensitive only to Colistin. Out of 13 patients from whom only Colistin sensitive Acinetobacter spp. was isolated, nine (69.23\%) and out of eight patients from whom only Colistin sensitive $P$. aeruginosa was isolated three (37.50\%) were elderly (age $\geq 65$ years)

\section{Table 1: Clinical sample and Acinetobacter/Pseudomonas isolates during study period}

\begin{tabular}{|llcccc}
\hline S.N. & Clinical sample & $\begin{array}{c}\text { Number of samples } \\
\text { received during the } \\
\text { study period }\end{array}$ & $\begin{array}{c}\text { Acinetobacter/ } \\
\text { Pseudomonas isolates } \\
\mathbf{n}(\%)\end{array}$ & $\begin{array}{c}\text { Acinetobacter } \\
\text { spp. Isolates } \\
\mathbf{n}(\%)\end{array}$ & $\begin{array}{c}\text { Pseudomonas } \\
\text { aeruginosa } \\
\text { isolates n (\%) }\end{array}$ \\
\hline $\begin{array}{l}\text { Respiratory (sputum, tracheal aspirate, } \\
\text { endotracheal tube, throat swab, etc.) }\end{array}$ & 1120 & $53(4.73)$ & $16(30.19)$ & $37(69.81)$ \\
\hline 2 & Urine & 5374 & $15(0.28)$ & $3(20)$ & $12(80)$ \\
\hline 3 & Pus/wound swab & 1088 & $18(1.65)$ & $6(33.33)$ & $12(66.67)$ \\
\hline 4 & Blood & 1973 & $1(0.05)$ & $1(100)$ & - \\
\hline 5 & Body fluid (ascitic fluid, bile fluid, etc.) & 150 & $5(3.33)$ & $1(20)$ & $4(80)$ \\
\hline
\end{tabular}

Table 2: Antibiotic sensitivity pattern of Pseudomonas aeruginosa and Acinetobacter species.

\begin{tabular}{|clcc}
\hline S.N. & Antibiotics & Pseudomonas (n=65) & Acinetobacter (n=27) \\
$\mathbf{n}(\%)$ & $9(33.33)$ \\
\hline 1 & Ciprofloxacin & $52(80)$ & $9(33.33)$ \\
\hline 2 & Levofloxacin & $53(81.54)$ & $9(33.33)$ \\
\hline 3 & Ceftazidime & $52(80)$ & $9(33.33)$ \\
\hline 4 & Cefotaxime & - & $9(33.33)$ \\
\hline 5 & Cefepime & $52(80)$ & $10(37.04)$ \\
\hline 6 & Cefoperazone-salbactum & - & $10(37.04)$ \\
\hline 7 & Piperacillin-tazobactum & $55(84.61)$ & $6(22.22)$ \\
\hline 8 & Gentamicin & $55(84.61)$ & $6(22.22)$ \\
\hline 9 & Amikacin & $55(84.61)$ & $14(51.85)$ \\
\hline 10 & Imipenem & $57(87.69)$ & $14(51.85)$ \\
\hline 11 & Meropenem & $57(87.69)$ & $27(100)$ \\
\hline 12 & Colistin & $65(100)$ & \\
\hline
\end{tabular}


In this study, 23 (25\%) of the patients had prolonged hospital stay and $69(75.00 \%)$ of the patients had no prolonged hospital stay (less than two weeks). Number of patients with prolonged hospital stay from whom Acinetobacter spp. was isolated 14 (60.87\%) and $P$. aeruginosa was nine (39.13\%). In the present study, 22 (95.65\%) multidrug-resistant (MDR) bacteria were isolated from prolonged hospital stayers compared to only nine $(13.04 \%)$ MDR bacteria isolated from the patients who had no prolonged hospital stay ( $\mathrm{p}$-value $<0.001)$. In this study $16(94.12 \%)$ out of the total 17 diabetic patients number had MDR bacteria isolates which was much more in comparison to only $15(20 \%)$ MDR bacterial isolates out of total 75 non-diabetic patients ( $p$-value $<0.001$ ). In the current study 31 (33.69\%) were elderly patients (age $\geq 65$ years) and 61 (66.31\%) were of age $<65$ years old. Seventeen (54.84\%) of elderly patients had MDR isolates whereas only $14(22.95 \%)$ of patients who were not elderly had MDR isolates ( $\mathrm{p}$-value $<0.0047)$.

\section{DISCUSSION}

The present study detected Acinetobacter spp. and $P$. aeruginosa isolates from various clinical samples and observed the antibiotic susceptibility pattern along with few predisposing factors. Total number of clinical samples received during the study period was 9,705 and out of this the total numbers of Acinetobacter spp. and $P$. aeruginosa isolates were $0.95 \%$. This finding is much less than the finding in the study conducted by Mirzaei et al. in Iran in which out of 3,248 clinical samples, $A$. baumannii and $P$. aeruginosa strains were detected in $9.51 \%$ of samples. ${ }^{9}$ Most of the sample from which isolates were found was of respiratory tract origin which was $57.61 \%$ and $59.26 \%$ of Acinetobacter spp. isolates and $56.92 \%$ if $P$. aeruginosa isolates were detected in respiratory samples which is similar but lesser in number in comparison to the finding in the study conducted by Baral et al. in western part of Nepal from 2014 to 2016 in which $74.7 \%$ of Acinetobacter spp. and $65.8 \%$ of $P$. aeruginosa were of respiratory tract origin. ${ }^{3}$ The reason for present study having lesser percentage compared to that study may be because their study duration was three years and this was only one year. Total number of Acinetobacter spp. isolates was $29.35 \%$ and $P$. aeruginosa isolates was $70.65 \%$. Percentage wise this finding agrees with the finding in the study conducted by Baral et al. in which out of 483 isolates $35.20 \%$ were Acinetobacter spp. isolates and $64.80 \%$ were $P$. aeruginosa isolates. ${ }^{3}$

Antibiotic susceptibility pattern of $P$. aeruginosa showed that, $80 \%$ of the strains were sensitive to Ciprofloxacin,
Ceftazidime, Cefepime; and $81.54 \%$ sensitive to Levofloxacin and $84.61 \%$ sensitive to Piperacillintazobactum, Gentamicin and Amikacin. This finding seems quite close with the finding of the study done by Nepal et al. in 2016 in which Gentamicin, Amikacin and Piperacillin-tazobactum were more than $80 \%$ sensitive. ${ }^{10}$ In present study, $87.69 \%$ were sensitive to carbapenem this agrees with the finding in the study conducted by Mishra et al. in 2008, in which Carbapenem sensitivity was observed more than $80 \% .{ }^{11}$ Carbapenems are used as last option for treatment $P$. aeruginosa infections and other Gram-negative bacterial infections. ${ }^{12,13}$ Carbapenemresistant $P$. aeruginosa has become prevalent in our setting too just as globally. ${ }^{14,15}$ Colistin seemed to be the best antibiotic for the treatment of $P$. aeruginosa infection in present study as all strains included in this study were sensitive to it and it may be useful drug when choices are limited. Colistin might be a useful drug to treat carbapenem resistant strains and this agrees with the findings of Sabuda et al. between 2000 to 2005. ${ }^{16}$

Antibiotic susceptibility pattern of Acinetobacter spp. showed that only $22.22 \%$ the strains were sensitive to Gentamicin and Amikacin which correlates with the findings of Baniya et al. ${ }^{17}$ Problem of aminoglycoside resistance seems to be a major concern in the current study setting, which agrees with the finding in the study conducted by Moniri et al. in Iran. ${ }^{18}$ Less than $40 \%$ of the Acinetobacter spp. were sensitive to Ciprofloxacin, Levofloxacin, Ceftazidime, Cefotaxime, Cefepime, Cefoperazone-salbactum and Piperacillin-tazobactum which agrees with the findings of Moniri et al., in which more than $60 \%$ Acinetobacter spp. were resistant to ceftazidime, ciprofloxacin, levofloxacin, piperacillin/ tazobactam. ${ }^{18}$ Less sensitivity of Acinetobacter spp. to fluoroquinolones and beta-lactam antibiotics seems another problem in current study setting, similar to other studies conducted in Nepal and outside Nepal. ${ }^{17,19}$ Only $51.85 \%$ of Acinetobacter spp. were sensitive to carbapenem which is little less than the findings in the study conducted by Baniya et al. in which it was $56 \%$ sensitive, but much more than the findings in the study conducted by Yadav et al. in which carbapenem sensitivity was only around $20 \% \cdot{ }^{17,20}$

Since, all Acinetobacter spp. were sensitive to Colistin it seemed to be the best drug for the treatment of any infection caused by Acinetobacter spp. In the study conducted by Yadav et al. too all strains of Acinetobacter spp. were susceptible to Colistin but in the study conducted in other parts of Nepal by Baniya et al. and Raut et al., Acinetobacter spp. was not fully susceptible to Colistin, but it was $74 \%$ and $71.4 \%$ respectively. ${ }^{17,20,21}$ This 
shows that in future there is a high risk that current study setting also might come across these types of strains.

Over all $33.69 \%$ of the isolates were MDR and multidrug resistance was seen among much more among Acinetobacter spp. in comparison to $P$. aeruginosa ( $66.67 \%$ vs. $20.00 \%)$. In the study conducted by Baral et al. too, Acinetobacter spp. seemed to be more multidrugresistant in comparison to $P$. aeruginosa $(75.9 \%$ vs. $60.1 \%){ }^{3}$ Similar finding was observed in the study conducted by Mirzaei et al. in Iran. ${ }^{9}$ Uncontrolled use of broad-spectrum antibiotics including carbapenem might be a major cause for the global rise in MDR Acinetobacter spp. ${ }^{22}$ In total, $12.31 \%$ P. aeruginosa and $48.15 \%$ Acinetobacter spp. were Colistin-only-sensitive. Colistinonly-sensitive $P$. aeruginosa and Acinetobacter spp. from ICU patients has been reported in several studies. ${ }^{23,24}$ Colistin seems to be effective to treat infection caused by multidrug-resistant $P$. aeruginosa and Acinetobacter spp. in this setting and elsewhere too. ${ }^{25}$

Of all, $69.23 \%$ of the patients were elderly from whom Colistin-only-sensitive Acinetobacter spp. was isolated and only $37.50 \%$ of the patients from whom Colistinonly-sensitive $P$. aeruginosa strain was isolated were elderly.

In this study, $25 \%$ of the patients had prolonged hospital stay and $75 \%$ of the patients had no prolonged hospital stay. Among them, $60.87 \%$ of Acinetobacter spp. and only $39.13 \%$ of $P$.aeruginosa strains were isolated from patients who had prolonged hospital stay. Similarly, 95.65\% MDR bacteria was isolated from prolonged hospital stayers compared to only $13.04 \%$ MDR bacteria isolated from the patient who had no prolonged hospital stay ( $p$-value $<0.001$ ). Hence, in this study prolonged hospital stay might be a predisposing factor for increased incidence of MDR pathogens which agrees with the findings of Baral et al. in which the hospital stay was longer for patients

\section{REFERENCES}

1. Cerceo E, Deitelzweig SB, Sherman BM, Amin AN. Multidrug-resistant gram-negative bacterial infections in the hospital setting: Overview, implications for clinical practice, and emerging treatment options. Microb Drug Resist. 2016 Jul;22(5):412-31. [PubMed | Full Text | DOI]

2. Baral S, Pokharel A, Subramanya SH, Nayak N. Clinico-epidemiological profile of Acinetobacter infected with MDR isolate ( $p=0.001$ for Acinetobacter spp. and $p=0.003$ for $P$. aeruginos $a)^{3}$

In this study $94.12 \%$ of diabetic patients had MDR bacteria isolates and only $20 \%$ of non-diabetic patients had MDR isolates ( $p$-value $<0.001$ ). Hence, in this study diabetes seems to be another predisposing factor for incidence of MDR pathogens and diabetes mellitus is an important risk factor for colonisation with MDR Acinetobacter spp. as detected in the study conducted by Mody et al. in $2015 .{ }^{26}$ In present study, $54.84 \%$ of elderly patients had MDR isolates whereas only $22.95 \%$ of patients who were not elderly had MDR isolates ( $p$-value $=0.0047$ ). Hence, in current study old age seems to be another predisposing factor for developing antimicrobial resistance and elderly patients have to be taken proper care to prevent this. Similar finding was observed in the study conducted by Pappas et al. in 2009. ${ }^{27}$

\section{CONCLUSION}

In this study Acinetobacter spp. and Pseudomonas aeruginosa strains were isolated from various clinical samples and mainly from respiratory samples. Multidrug resistance and predisposing factors such as length of hospital stay, diabetes, and old age have become a major concern. Management of such infection in the community as well as hospital by early investigation and analysis of infection and controlling of risk factors might help to reduce the burden of respiratory tract infection in health care centres and communities.

\section{ACKNOWLEDGMENTS}

We express our profound gratitude to the Department of Microbiology and Department of Internal Medicine, Kathmandu University Hospital for their kind support and cooperation throughout this study.

\section{Conflict of interest: None Source(s) of support: None}


4. Rice LB. Federal funding for the study of antimicrobial resistance in nosocomial pathogens: no ESKAPE. J Infect Dis. 2008 Apr 15;197(8):1079-81. [PubMed | Full Text | DOI]

5. Clinical and Laboratory Standards Institute. Performance standards for antimicrobial susceptibility testing. 28th ed. CLSI supplement M100. Wayne, PA: CLSI; 2018. [Full Text]

6. Antimicrobial-Resistant Phenotype Definitions. Analysis of antimicrobial-resistant organisms in NHSN updated 2021. Atlanta, GA: Centers for Disease Control and Prevention; 2021. [Full Text]

7. Moradiya Y, Murthy S, Shah S, Modi S. Risk factors and outcomes of prolonged hospitalization after intracerebral hemorrhage in the United States. Neurology. 2014 April ;82(10 Suppl)P7.140. [Full Text]

8. Singh S, Bajorek B. Defining 'elderly' in clinical practice guidelines for pharmacotherapy. Pharm Pract (Granada). 2014;12(4):489-97. [PubMed | Full Text | DOI]

9. Mirzaei, B, Bazgir, ZN, Goli, HR, Iranpour F, Mohammadi F, Babaei R. Prevalence of multi-drug resistant (MDR) and extensively drug-resistant (XDR) phenotypes of Pseudomonas aeruginosa and Acinetobacter baumannii isolated in clinical samples from Northeast of Iran. BMC Res Notes. 2020 Aug 10;13(1):380. [PubMed | Full Text | DOI]

10. Nepal R, Shrestha B, Joshi DM, Joshi RD, Shrestha $\mathrm{S}$, Singh A. Antibiotic Susceptibility Pattern of Gram-negative Isolates of Lower Respiratory Tract Infection. J Nepal Health Res Counc. 2018 Mar 13;16(1):22-6. [PubMed | Full Text | DOI]

11. Mishra SK, Acharya J, Kattel HP, Koirala J, Rijal BP, Pokhrel BM. Metallo-beta-lactamase producing gram-negative bacterial isolates. J Nepal Health Res Counc. 2012 Sep;10(22):208-13. [PubMed | Full Text]

12. Giamarellou H, Poulakou G. Multidrug-resistant Gram-negative infections: What are the treatment options? Drugs. 2009 Oct 1;69(14):1879-901. [PubMed | Full Text | DOI]

13. Riera E, Cabot G, Mulet X, García-Castillo M, Campo R, Juan $C$, et al. Pseudomonas aeruginosa carbapenem resistance mechanisms in Spain: impact on the activity of imipenem, meropenem and doripenem. J Antimicrob Chemother. 2011 Sep;66(9):2022-7. [PubMed | Full Text | DOI]

14. Nagao $M$, linuma $Y$, Igawa J, Saito $T$, Yamashita $\mathrm{K}$, Kondo T, et al. Control of an outbreak of carbapenem-resistant Pseudomonas aeruginosa in a haemato-oncology unit. J Hosp Infect. 2011 Sep;79(1):49-53. [PubMed | Full Text | DOI]
15. Livermore DM. Has the era of untreatable infections arrived? J Antimicrob Chemother. 2009 Sep;64 Suppl 1:i29-36. [PubMed] [Full Text | DOI]

16. Sabuda DM, Laupland K, Pitout J, Dalton B, Rabin H, Louie $T$, et al. Utilisation of colistin for treatment of multidrug-resistant Pseudomonas aeruginosa. Can J Infect Dis Med Microbiol. 2008 Nov;19(6):413-8. [PubMed | Full Text | DOI]

17. Baniya B, Pant ND, Neupane S, Khatiwada S, Yadav UN, Bhandari N, et al. Biofilm and metallo beta-lactamase production among the strains of Pseudomonas aeruginosa and Acinetobacter spp. at a tertiary care hospital in Kathmandu, Nepal. Ann Clin Microbiol Antimicrob. 2017 Nov 2;16(1):70. [PubMed | [Full Text | DOI]

18. Moniri R, Farahani RK, Shajari G, Shirazi MN, Ghasemi A. Molecular epidemiology of aminoglycosides resistance in Acinetobacter spp. with emergence of multidrug-resistant strains. Iran J Public Health. 2010;39(2):63-8. [PubMed | Full Text]

19. Solomon FB, Wadilo F, Tufa EG, Mitiku M. Extended spectrum and metalo beta-lactamase producing airborne Pseudomonas aeruginosa and Acinetobacter baumanii in restricted settings of a referral hospital: A neglected condition. Antimicrob Resist Infect Control. 2017 Oct 23;6:106. [PubMed | Full Text | DOI]

20. Yadav SK, Bhujel R, Mishra SK, Sharma S, Sherchand JB. Emergence of multidrug-resistant nonfermentative gram negative bacterial infection in hospitalised patients in a tertiary care center of Nepal. BMC Res Notes. 2020 Jul 2;13(1):319. [PubMed | Full Text | DOI]

21. Raut S, Rijal KR, Khatiwada S, Karna S, Khanal $\mathrm{R}$, Adhikari J, et al. Trend and characteristics of Acinetobacter baumannii infections in patients attending Universal College of Medical Sciences, Bhairahawa, western Nepal: A longitudinal study of 2018. Infect Drug Resist. 2020 Jun 8;13:1631-41. [PubMed | Full Text | DOI]

22. Van TD, Dinh QD, Vu PD, Nguyen TV, Pham CV, Dao $\Pi$, et al. Antibiotic susceptibility and molecular epidemiology of Acinetobacter calcoaceticusbaumannii complex strains isolated from a referral hospital in northern Vietnam. J Glob Antimicrob Resist. 2014 Dec;2(4):318-21. [PubMed | Full Text | DOI]

23. Mastoraki A, Douka E, Kriaras I, Stravopodis G, Manoli H, Geroulanos S. Pseudomonas aeruginosa susceptible only to colistin in intensive care unit patients. Surg Infect (Larchmt). 2008 Apr;9(2):15360. [PubMed | Full Text | DOI] 
24. Jacob I, Rangappa P, Thimmegowda LC, Rao K. A study of multidrug-resistant, colistin-only-sensitive infections in intubated and mechanically ventilated patients over 2 years. J Glob Infect Dis. 2020 Feb 19;12(1):5-10. [PubMed | Full Text | DOI]

25. Lu Q, Luo R, Bodin L, Yang J, Zahr N, Aubry A, et al. Efficacy of high-dose nebulised colistin in ventilatorassociated pneumonia caused by multidrugresistant Pseudomonas aeruginosa and Acinetobacter baumannii. Anesthesiology. 2012 Dec;117(6):133547. [PubMed | Full Text | DOI]
26. Mody L, Gibson KE, Horcher A, Prenovost K, McNamara SE, Foxman B, et al. Prevalence of and risk factors for multidrug-resistant Acinetobacter baumannii colonisation among high-risk nursing home residents. Infect Control Hosp Epidemiol. 2015;36(10):1155-62. [PubMed | Full Text | DOI]

27. Pappas G, Saplaoura K, Falagas, ME. Current treatment of pseudomonal infections in the elderly. Drugs Aging. 2009;26(5):363-79. [PubMed | Full Text | DOI] 